\title{
Brain Connectivity Associated with Muscle Synergies in Humans
}

\author{
@Manku Rana, ${ }^{1}{ }^{\circledR}$ Moheb S. Yani, ${ }^{1}$ Skulpan Asavasopon, ${ }^{2}$ Beth E. Fisher, ${ }^{1,3}$ and ${ }^{-J a s o n ~ J . ~ K u t c h ~}{ }^{1}$ \\ ${ }^{1}$ Division of Biokinesiology and Physical Therapy, University of Southern California, Los Angeles, California 90033, ${ }^{2}$ Physical Therapy Department, Loma \\ Linda University, Loma Linda, California 92350, and ${ }^{3}$ Department of Neurology, University of Southern California, Los Angeles, California 90033
}

The human brain is believed to simplify the control of the large number of muscles in the body by flexibly combining muscle coordination patterns, termed muscle synergies. However, the neural connectivity allowing the human brain to access and coordinate muscle synergies to accomplish functional tasks remains unknown. Here, we use a surprising pair of synergists in humans, the flexor hallucis longus (FHL, a toe flexor) and the anal sphincter, as a model that we show to be well suited in elucidating the neural connectivity underlying muscle synergy control. First, using electromyographic recordings, we demonstrate that voluntary FHL contraction is associated with synergistic anal sphincter contraction, but voluntary anal sphincter contraction occurs without FHL contraction. Second, using fMRI, we show that two important medial wall motor cortical regions emerge in relation to these tasks: one located more posteriorly that preferentially activates during voluntary FHL contraction and one located more anteriorly that activates during both voluntary FHL contraction as well as voluntary anal sphincter contraction. Third, using transcranial magnetic stimulation, we demonstrate that the anterior region is more likely to generate anal sphincter contraction than FHL contraction. Finally, using a repository resting-state fMRI dataset, we demonstrate that the anterior and posterior motor cortical regions have significantly different functional connectivity with distinct and distant brain regions. We conclude that specific motor cortical regions in humans provide access to different muscle synergies, which may allow distinct brain networks to coordinate muscle synergies during functional tasks.

Key words: EMG; fMRI; functional connectivity; motor cortex; pelvic floor; TMS

\section{Significance Statement}

How the human nervous system coordinates activity in a large number of muscles is a fundamental question. The brain and spinal cord are believed to simplify the control of muscles by grouping them into functional units called muscle synergies. Motor cortex is involved in activating muscle synergies; however, the motor cortical connections that regulate muscle synergy activation are unknown. Here, we studied pelvic floor muscle synergies to elucidate these connections in humans. Our experiments confirmed that distinct motor cortical regions activate different muscle synergies. These regions have different connectivity to distinct brain networks. Our results are an important step forward in understanding the cortical control of human muscles synergies, and may also have important clinical implications for understanding movement dysfunction.

\section{Introduction}

Controlling muscles in groups, termed muscle synergies, has been proposed as an important strategy used by the nervous sys-

Received May 21, 2015; revised Sept. 18, 2015; accepted Sept. 26, 2015.

Author contributions: M.R., M.S.Y., S.A., B.E.F., and J.J.K. designed research; M.R., M.S.Y., and S.A. performed research; M.R., M.S.Y., and J.J.K. analyzed data; M.R., M.S.Y., S.A., B.E.F., and J.J.K. wrote the paper.

The contents of this research paper were developed in part under support from the University of Southern California Division of Biokinesiology and Physical Therapy, Loma Linda University Physical Therapy Department, and National Center for Medical Rehabilitation Research of the National Institutes of Health under Award T32 HD064578. The contents do not necessarily represent the official views of the National Institutes of Health. We thank Ms. Harjot Hansra for assistance with data collection, and all of the research participants for their time.

The authors declare no competing financial interests.

Correspondence should be addressed to Dr. Jason J. Kutch, University of Southern California, 1540 E. Alcazar Street, CHP 155, Los Angeles, CA 90033. E-mail: kutch@usc.edu. tem to simplify the control of $>600$ muscles in the human body (Bernstein, 1967; Bizzi et al., 1991; Ting and Macpherson, 2005; Torres-Oviedo et al., 2006; Giszter et al., 2007). Each muscle synergy generates a particular mechanical action, and the flexible combination of muscle synergies is thought to allow a wide repertoire of natural motor behaviors (Giszter et al., 1993; MussaIvaldi et al., 1994b; Kargo and Giszter, 2000; d'Avella et al., 2003).

Muscle synergies are defined to be consistent patterns of spatiotemporal muscle activity (d'Avella et al., 2003; Ting and Macpherson, 2005) that are elicited by activation of specific neural centers (Saltiel et al., 2001). In this paper, we refer to these 
neural centers that active muscle synergies as "synergy access points." Muscle synergies may be structured in spinal circuitry (Mussa-Ivaldi et al., 1994a; Saltiel et al., 2001; Cheung et al., 2009) and are activated by supraspinal regions, such as motor cortex (Drew et al., 2008; Overduin et al., 2014; Waters-Metenier et al., 2014). However, the brain networks that enable flexible combinations of muscle synergies to generate movements have not been identified in humans. We hypothesized that the specific regions in the motor cortex can activate different muscle synergies, and these synergy access points connect with distinct brain regions encoding different task demands. This architecture would allow flexible coordination of muscle synergies based on the task.

We used pelvic floor muscle synergies to test this hypothesis in humans because we have previously shown that these synergies involve cortical activation (Asavasopon et al., 2014). Pelvic floor muscles contract automatically with trunk and lower limb muscles that are distinct from the pelvic floor as follows: shoulder muscles (Hodges et al., 2007), gluteal muscles (Bø and Stien, 1994), abdominal muscles (Hodges et al., 2007), and toe muscles (Sapsford et al., 2010), presumably to promote continence and provide spinal support during functional tasks. However, lower limb muscles need not be activated during voluntary pelvic floor muscle contraction (Bø and Stien, 1994; Schrum et al., 2011; Asavasopon et al., 2014), suggesting that lower limb muscles and pelvic floor muscles are recruited as part of at least two different muscle synergies. We therefore hypothesized that there is a motor cortical synergy access point that more directly activates pelvic floor muscles compared with pelvic floor synergists, and that this access point preferentially connects to brain regions known to be involved in continence control (Fowler et al., 2008; Tadic et al., 2012).

The goal of the present study was to expand on our prior work (Asavasopon et al., 2014) by examining the specificity of how motor cortical regions activate different target muscles when stimulated, and how motor cortical regions are functionally connected to the rest of the brain. Although our previous work examined the automatic coactivation of pelvic floor muscles and gluteal muscles, we found that studying the synergistic coupling between pelvic floor muscles and toe muscles provided a model system better suited to the goals of the present study (see Discussion). The results to be described below show that the anal sphincter and toe muscles are controlled by at least two muscle synergies that are activated by distinct regions in motor cortex. We then demonstrate that these motor cortical regions differentially connect with distant and distinct brain regions. To our knowledge, our current study is the first to use resting-state functional connectivity to demonstrate how large-scale human brain networks might interact with motor cortex to allow flexible combination of muscle synergies based on task demands.

\section{Materials and Methods}

Participant population. We recruited 16 healthy men with a mean age ( $\pm \mathrm{SD}$ ) of $32.6 \pm 6.0$ years (range, $24-43$ years). The studies we describe here with these individuals were performed at the University of Southern California and approved by the University of Southern California Institutional Review Board. All participants provided informed consent. The inclusion criteria for the study were as follows: the participants had to be older than 18 years, have no history of neurological conditions, and be able to participate in the informed consent process. The exclusion criteria as follows: history of significant head trauma; electrical, magnetic, or mechanical implantation; history of seizures; immediate family member with epilepsy; current abuse of alcohol or drugs; anticipated inability to complete the study; or history of hallucinations or schizophrenia.
Here we describe a series of studies using EMG, fMRI during muscle contraction, transcranial magnetic stimulation (TMS), and resting-state fMRI. The EMG study defined patterns of muscle coordination and was performed first. The fMRI study of muscle contraction was performed next and defined brain ROI associated with different muscle coordination patterns. The TMS study then followed to define the specificity of muscle activation elicited by stimulation of the fMRI-identified ROI. Finally, a resting-state fMRI analysis of the functional connectivity of the identified ROI was performed on a repository resting-state fMRI dataset. Participant numbers varied across the different studies and are indicated below.

EMG acquisition and analysis. We acquired and analyzed EMG data as described previously for the analysis of pelvic floor muscle synergies (Asavasopon et al., 2014), which are described briefly here. In 10 participants, we measured muscle activity using EMG to define the characteristics of anal sphincter synergies before performing the same tasks using fMRI to define the neural substrate of these synergies. We used EMG to verify the muscle synergy between the anal sphincter and flexor hallucis longus muscle (FHL, a flexor of the great toe) and to establish finger muscle contraction as an appropriate control muscle group that does not have synergistic coupling with the anal sphincter. We recorded surface EMG data from the right FHL, the anal sphincter, and the right first dorsal interosseous muscle (FDI) while the participants were lying supine in a mock MRI scanner. We recorded EMG signals from the FHL and FDI with miniature electrode/preamplifiers (Delsys) with two recording surfaces, $5 \mathrm{~mm}$ long and $10 \mathrm{~mm}$ apart. We recorded an EMG signal from the anal sphincter using a medical-grade rectal EMG sensor (Prometheus Group), which provided a bipolar recording from two bar electrodes, 12 $\mathrm{mm}$ apart, with dimensions $30 \mathrm{~mm} \times 7 \mathrm{~mm}$, mounted longitudinally along a cylindrical plug-type applicator. The EMG preamplifier filters had a bandwidth of $20-450 \mathrm{~Hz}$, with gains of 1000 for FHL and FDI, 10,000 for anal sphincter, and a sampling rate of $2000 \mathrm{~Hz}$.

Before the experimental session, we asked participants to empty their bladder. Participants performed separate trials, each of which involved repeated voluntary contractions of a different primary muscle group. In anal sphincter trials, we instructed participants to contract their pelvic floor as if to maintain continence. In FHL trials, we instructed participants to generate right toe flexion. In FDI trials, we instructed participants to contract their FDI muscle to generate index finger abduction. For all trial types, we first acquired EMG data corresponding to maximal voluntary contraction (MVC). Because we planned to repeat voluntary contraction trials in the fMRI scanner without EMG, we instructed participants to produce moderate muscle contraction ( $\sim 20 \%$ effort) to avoid fatigue during the EMG portion of the study that was performed in the mock MRI. EMG activity was quantified and expressed as percentage MVC.

We first estimated the contraction onsets of the primary muscle group under voluntary control in each trial and then determined whether timelocked EMG changes occurred in other recorded muscles. To perform this analysis, EMG signals from all recorded muscles were first high-pass filtered at $100 \mathrm{~Hz}$ (fourth-order zerolag Butterworth filter), rectified, low-pass filtered at $30 \mathrm{~Hz}$ (Hodges et al., 2007), and then normalized to identically processed EMG data from the maximum contraction trial. Contraction onsets were defined to occur when the smoothed EMG exceeded 2 SDs of the EMG baseline noise with the muscle at rest. Within each participant, we then defined an EMG transient for each muscle and each trial by averaging the rectified and filtered EMG data across repeated muscle contractions within a time window spanning $1 \mathrm{~s}$ before to $3 \mathrm{~s}$ after the contraction onset of the primary muscle for the trial. To define significant EMG magnitude changes, we performed group statistics on the maximum of the EMG transient for muscles of interest within each participant. We defined synergistic anal sphincter muscle activity to have occurred with voluntary FHL muscle activation if the anal sphincter EMG magnitude was significantly greater during voluntary FHL activation compared with during voluntary FDI activation. We used FDI as a reference because we have previously shown that voluntary FDI activation does not generate synergistic pelvic floor muscle activation (Asavasopon et al., 2014).

fMRI acquisition and analysis. In 14 participants, we measured brain activation associated with the voluntary muscle contraction tasks (de- 
scribed above) using fMRI as we have described previously (Asavasopon et al., 2014). Briefly, we used a 3 tesla (GE Signa Excite) with an 8-channel head coil. We positioned participants supine viewing a fixation crosshair and placed foam pads to limit head motion. We collected T2-weighted echo planar image volumes with BOLD contrast (echo time $34.5 \mathrm{~ms}$, flip angle 90 degrees, field of view $220 \mathrm{~mm}$, pixel size $3.43 \mathrm{~mm}$ ) continually every $2.5 \mathrm{~s}$ during three imaging runs. Each volume consisted of 37 axial slices ( $3 \mathrm{~mm}$ slice thickness, $0.5 \mathrm{~mm}$ interslice gaps) that covered the brain from vertex to cerebellum. We additionally acquired a T1-weighted high-resolution anatomical image from each participant. We cued participants to voluntarily contract each muscle group as during EMG acquisition (to 20\% effort) in three separate runs (anal sphincter contraction run, FHL contraction run, and FDI contraction run) as described above for the EMG experiments, with the exception that participants performed additional contraction blocks ( 6 blocks of 10 contractions) in the scanner. All 14 participants performed anal sphincter contraction runs and FHL contraction runs, and 10 participants performed FDI contraction runs.

We preprocessed each participant's fMRI data using the FMRIB Expert Analysis Tool (http://fsl.fmrib.ox.ac.uk/fsl/fslwiki/), which included skull extraction using the brain extraction tool in FSL, slice timing correction, motion correction, spatial smoothing using a Gaussian kernel with FWHM of $5 \mathrm{~mm}$, and nonlinear high-pass temporal filtering (100 s). We used a GLM to examine the changes in BOLD signal associated with muscle contraction for the three tasks. We performed participant-level whole-brain GLM analyses of individual runs in each participant to determine the change in BOLD signal during the contraction blocks compared with the rest blocks. We then performed a group-level mixed-effect (FLAME 1 in FSL) analysis, with unpaired two-sided $t$ tests, to identify voxels in standard MNI coordinates with significant differences in response based on the muscle group being voluntary contracted by the participant. We thresholded group-level images with cluster-based correction for multiple comparisons with $Z>2.3$ and $p<0.05$. Significant voxels with a greater probability of being part of precentral gyrus compared with postcentral gyrus, as defined by the Harvard-Oxford Cortical Structural Atlas in FSL, were defined as motor ROIs.

TMS acquisition and analysis. In five participants, we obtained motorevoked potentials (MEPs) from the anal sphincter and FHL, with participants resting supine, using a single-pulse magnetic stimulator (Magstim $200^{2}$, Magstim) with a $110 \mathrm{~mm}$ double-cone coil. We sampled EMG signal at $16,000 \mathrm{~Hz}$, bandpass filtered at $1-1000 \mathrm{~Hz}$, and amplified at a gain of 9500. In our previous study of pelvic floor MEPs, we stimulated along the midline and monitored pelvic floor MEP amplitude as we passed over the pelvic floor motor representation (Asavasopon et al., 2014). In the current study, to examine the specificity of motor cortical stimulation response in multiple lower limb muscles, we used our fMRIidentified motor cortical ROI to focus stimulation on two target locations. We constructed participant-specific stimulation grids covering the ROI identified in the group fMRI activation statistics associated with anal sphincter and FHL voluntary contractions. As described in Results, the motor cortical region activated during FHL contractions was divided into two ROI, the anterior motor ROI (A-motor) and posterior motor ROI (P-motor). The A-motor ROI was active during both anal sphincter and FHL contraction, and the P-motor ROI was more active during FHL contraction compared with during anal sphincter contraction. We constructed grids with spatial resolution of $5 \times 5 \mathrm{~mm}$ using the Brainsight Frameless system (Rogue Research) to cover both A-motor and P-motor ROIs. We then stimulated the grid points in the two regions to obtain the anal sphincter hotspot over A-motor and the FHL hot spot over P-motor. We obtained input-output curves for both anal sphincter and FHL muscles based on stimulation of the anal sphincter hotspot over the A-motor
B Voluntary ASM Voluntary FHL

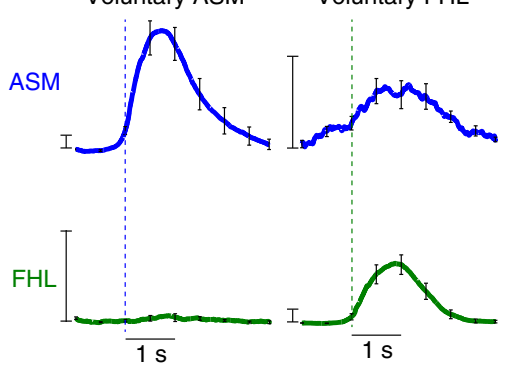

Figure 1. EMG evidence of anal sphincter and FHL synergy. EMG signals from the anal sphincter muscle (ASM; blue), and the FHL (green) muscle were recorded during separate trials that focused on the voluntary contraction of each of these muscles. $A$, Example contraction during voluntary FHL muscle contraction, but no FHL muscle contraction during voluntary ASM contraction. $B$, Group data demonstrating the consistent finding of synergistic contraction of the ASM during voluntary FHL. We did not find evidence of FHL muscle contraction during voluntary ASM contraction. Curves indicate the average EMG transient triggered by the onset of the task, averaged across participants. Error bars indicate SEM across participants. Scale bars, 2\% MVC

ROI and the FHL hotspot over the P-motor ROI. The stimulation intensities were chosen from a range with the minimum as the intensity that led to no observable MEP in muscles at their respective hotspots and the maximum based on the participant's comfort level. The maximum stimulation intensity ranged between $60 \%$ and $75 \%$ maximum stimulator output across all the participants. Stimuli were applied in random order.

We calculated MEP magnitude as the peak-to-peak magnitude of the EMG response to the stimulation in the time window of $10-100 \mathrm{~ms}$ after TMS pulse onset. The time window was selected to capture MEPs from both anal sphincter and FHL muscles (Pelliccioni et al., 1997; Kurusu and Kitamura, 1999; Turnbull et al., 1999; Lefaucheur, 2005). To combine across the participants, we expressed normalized MEP magnitudes as multiples $(>1)$ of the average peak-to-peak EMG, in the same time window relative to the TMS pulse, obtained from the trials that did not generate a MEP in response to the stimulation. Stimulation intensity was normalized to the resting motor threshold in each participant, using anal sphincter MEP for A-motor stimulation and FHL MEP for P-motor stimulation. Stimulation intensity was then binned into $10 \%$ bins $(100 \%-110 \%$ threshold, $110 \%-120 \%$ threshold, etc.). For group analysis, we used a two-way ANOVA (MATLAB, The MathWorks) to test the effect of stimulation intensity bin and cortical region (A-motor vs P-motor) on the normalized MEP magnitude in the two muscles.

Functional connectivity analysis of resting data. We performed functional connectivity analyses on a set of resting-state fMRI images from 48 participants from the 1000 Functional Connectome Project (http://www. nitrc.org/ir/). We selected 48 participants based on age and sex matching with the 14 participants recruited in our fMRI experiments. Data were preprocessed according to methods described previously (Roy et al., 2009; Baliki et al., 2012). At the individual-participant level, we then performed a functional connectivity analysis by contrasting the wholebrain connectivity of signals derived from the A-motor and P-motor ROIs, described above. We performed group-level analyses using FLAME 1 to obtain the average of the participant-level contrasts. We assessed significance with cluster-based corrections for multiple comparisons using Gaussian random field theory $(Z>2.3$; cluster significance: $p<0.05$, corrected).

\section{Results}

Using EMG recordings, we found that the anal sphincter and FHL coactivated during voluntary contraction of the FHL, but the anal sphincter activated by itself during voluntary contraction of the pelvic floor. Example recordings from one participant show that the anal sphincter activity was coupled with the activity of FHL during the task of FHL contraction, but this coupling did not exist for the voluntary task of anal sphincter contraction (Fig. 1A). Group data of time-locked anal sphincter EMG with FHL 
A
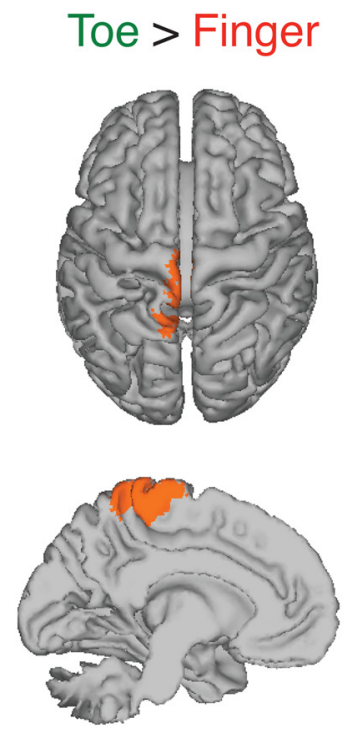

B
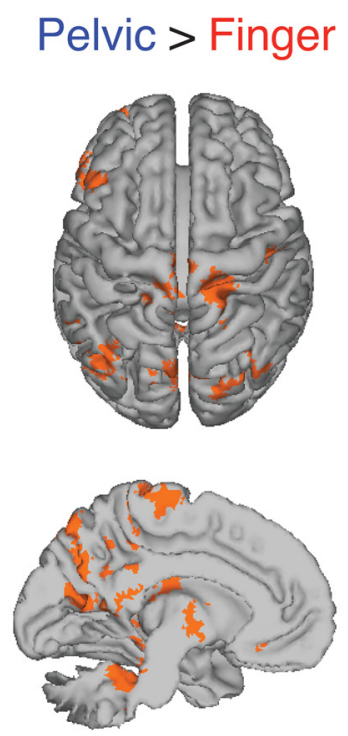

C
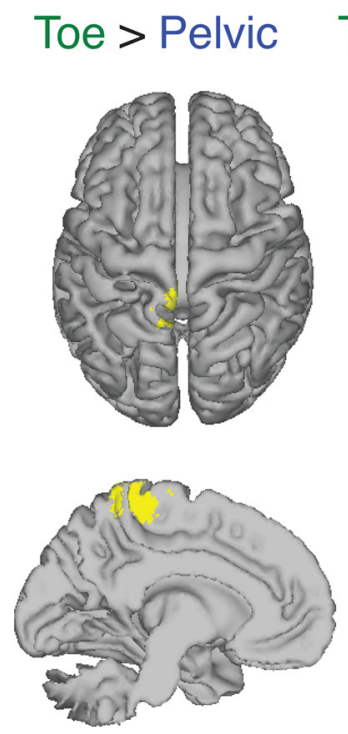

D

\section{Toe \& Pelvic > Finger}
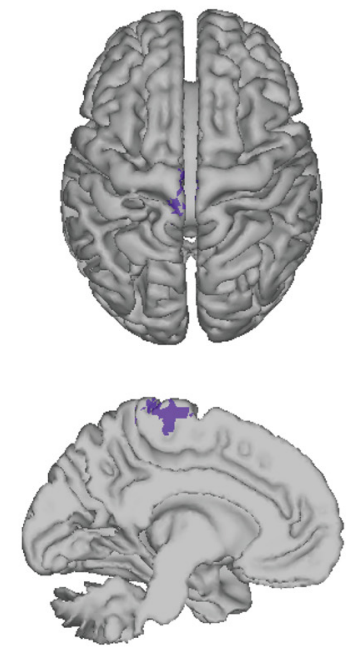

Figure 2. fMRl evidence of distinct regions involved in voluntary contraction of right FHL and anal sphincter (pelvic) synergy. fMRI data were collected while participants performed three separate runs of separate repeated voluntary contraction of pelvic floor muscles (PFM; Run 1), right FHL (Run 2), and right FDI (Run 3). $\boldsymbol{A}$, Contrast of voluntary FHL activation greater than voluntary FDI activation (toe $>$ finger) produced significant brain activation in medial motor cortex $(Z>2.3, p<0.05$, cluster corrected). $\boldsymbol{B}$, Contrast of voluntary PFM activation greater than voluntary FDI activation (pelvic $>$ finger) also produced significant activation in the medial motor cortex, which overlapped with the anterior portion of the significant activation region for toe $>$ finger $(Z>2.3$, $p<0.05$, cluster corrected). C, Posterior region of medial motor cortex exhibited significant brain activation, for voluntary FHL activation greater than anal sphincter (toe $>$ pelvic) $(Z>2.3, p<$ 0.05 , cluster corrected). $\boldsymbol{D}$, Anterior region of motor cortex exhibited significant brain activation, for both anal sphincter activation and FHL activation compared with FDl activation (toe and pelvic $>$ finger, conjunction of images in $A, B$ ).

showed that there was a consistent contraction of anal sphincter with the onset of FHL activity, but the change in time-locked FHL activity was not significant with the onset of anal sphincter activity (Fig. $1 B$ ). There was a consistent increase in time-locked anal sphincter activity (on an average, $1.2 \%$ of anal sphincter MVC) with FHL contraction that was significantly greater than the timelocked FHL activity with anal sphincter contraction (signed-rank test, $p=0.004)$. Furthermore, the time-locked anal sphincter activity with FHL contraction was significantly greater than that with the FDI contraction (signed-rank test, $p=0.002$ ). Even though the time-locked anal sphincter activity with FHL contraction was significantly greater than that with the FDI contraction, we still noticed a small amount of time-locked anal sphincter activity with voluntary FDI contraction (see Discussion). Anal sphincter activity was significantly greater during voluntary FDI activation compared with FDI activation during voluntary anal sphincter activation (signed-rank test, $p=0.002$ ).

Using fMRI (Fig. $2 A$ ), we found that the medial wall of precentral gyrus activated during the FHL contraction and anal sphincter contraction (Fig. 2A,B). The FHL region was composed of two functionally and anatomically distinct ROIs, anterior and posterior. The posterior ROI was obtained as the contrast of FHL activation greater than anal sphincter activation (Fig. 2C). The anterior ROI was obtained as the conjunction of the contrast of FHL activation greater than FDI activation and the contrast for anal sphincter activation greater than FDI activation (Fig. 2D), indicating activation during both voluntary anal sphincter contraction and voluntary FHL contraction. We found that both anterior ROI and posterior ROI contained contributions from the motor cortex; $74.7 \%$ of anterior ROI voxels were classified as motor cortex (A-motor) and $14.8 \%$ of posterior ROI voxels were classified as motor cortex (P-motor). The centroid of the motor cortical component of the A-motor in MNI coordinate system was $-2,-26,64 \mathrm{~mm}$, and that of the P-motor was -2 , $-32,60 \mathrm{~mm}$ (Table 1).

Using TMS, we found that the two regions, A-motor and P-motor, had different representations for anal sphincter and FHL. Example recordings from one participant show that the stimulation of anal sphincter hotspot over the A-motor ROI generated an MEP in the anal sphincter but not in the FHL. In contrast, stimulation over the P-motor ROI generated MEPs in both anal sphincter and FHL muscles (Fig. 3B). Example input-output curves for anal sphincter and FHL in response to stimulation of A-motor and P-motor indicate that the anal sphincter had similar response at both A-motor and P-motor while FHL generated MEPs only in response to stimulation of P-motor (Fig. 3C). Group data (Fig. 3D) indicate that there was a significant interaction of stimulus intensity and location for MEPs in the FHL $\left(F_{(2,21)}=24.17, p<0.0001\right)$. A post hoc test with Bonferroni correction for multiple comparisons indicated that larger MEPs were elicited in the FHL during stimulation of P-motor compared with A-motor at the stimulation intensity of $100 \%-110 \%(p<0.01)$, intensity of $110 \%-120 \%$ $(p<0.0001)$, and intensity of $120 \%-130 \%(p<0.0001)$. MEPs in the anal sphincter muscle were significantly affected by stimulation intensity $\left(F_{(2,24)}=27.06, p<0.0001\right)$, but there was no significant interaction between stimulus intensity and location $\left(F_{(2,24)}=2.63, p>0.05\right)$, indicating that stimulation of either A-motor or P-motor would evoke an approximately equal response in the anal sphincter.

Using the resting-state fMRI data, we found that the functional connectivity of the A-motor ROI was different from the functional connectivity of the P-motor ROI. This difference of functional connectivity occurred in five clusters for the contrast of A-motor connectivity greater than P-motor connectivity (Amotor > P-motor) and six clusters for the contrast of P-motor 
Table 1. Location, extent, and significance of the ROIs and each local maxima with altered functional connectivity of A-motor ROI with P-motor ROI

\begin{tabular}{|c|c|c|c|c|c|c|}
\hline \multirow[b]{2}{*}{ Region } & \multicolumn{3}{|c|}{ Coordinates (mm) } & \multirow[b]{2}{*}{ Cluster index } & \multirow[b]{2}{*}{ Cluster size } & \multirow[b]{2}{*}{$z$ scor } \\
\hline & $x$ & $y$ & $z$ & & & \\
\hline \multicolumn{7}{|l|}{ Centroid point } \\
\hline \multicolumn{7}{|l|}{ A-motor ROI } \\
\hline Pelvic $>$ finger & -2 & -26 & 64 & & 361 & 3.18 \\
\hline Toe $>$ finger & -2 & -26 & 64 & & & 4.02 \\
\hline \multicolumn{7}{|l|}{ P-motor ROl } \\
\hline Toe $>$ pelvic & -2 & -32 & 60 & & 92 & 3.74 \\
\hline \multicolumn{7}{|l|}{ Local maxima } \\
\hline \multicolumn{7}{|l|}{ A-motor $>$ P-motor } \\
\hline Precentral gyrus & -2 & -18 & 64 & 5 & 5930 & 10.1 \\
\hline SMA & 4 & 2 & 64 & 5 & & 5.06 \\
\hline Superior parietal lobule & 22 & -42 & 68 & 5 & & 4.68 \\
\hline Superior frontal gyrus & 20 & -10 & 66 & 5 & & 4.6 \\
\hline Superior temporal gyrus, right & 68 & -32 & 16 & 4 & 960 & 6.11 \\
\hline Central operculum cortex & 52 & -2 & 4 & 4 & & 3.9 \\
\hline Superior temporal gyrus, anterior & 66 & 0 & -4 & 4 & & 3.9 \\
\hline Superior temporal gyrus, posterior & 66 & -10 & -2 & 4 & & 3.8 \\
\hline Palanum temporal & 60 & -22 & 14 & 4 & & 3.65 \\
\hline Parietal operculum cortex, left & -50 & -26 & 14 & 3 & 701 & 4.55 \\
\hline Middle temporal gyrus, posterior division & -70 & -28 & 0 & 3 & & 3.43 \\
\hline Supramarginal gyrus & -62 & -30 & 22 & 3 & & 3.04 \\
\hline Planum polare & -54 & -6 & 2 & 2 & 638 & 4.14 \\
\hline Left insular cortex & -34 & 0 & 4 & 2 & & 3.52 \\
\hline Left putamen & -32 & -10 & 0 & 2 & & 3.42 \\
\hline Central opercular cortex & -44 & -2 & 4 & 2 & & 3.67 \\
\hline Inferior frontal gyrus & -60 & 12 & -4 & 2 & & 3.62 \\
\hline Temporal pole & -54 & 14 & -10 & 2 & & 3.04 \\
\hline Right insular cortex & 34 & -6 & 0 & 1 & 526 & 3.37 \\
\hline Right putamen & 30 & -14 & 4 & 1 & & 3.02 \\
\hline \multicolumn{7}{|l|}{ P-motor $>$ A-motor } \\
\hline Precuneous cortex & -20 & -64 & 22 & 6 & 6654 & 5.1 \\
\hline Cingulate gyrus, posterior division & 10 & -46 & 36 & 6 & & 4.76 \\
\hline Cuneal cortex & 2 & -76 & 26 & 6 & & 4.74 \\
\hline Angular gyrus & 58 & -48 & 18 & 6 & & 4.7 \\
\hline Paracingulate gyrus & 4 & 46 & 12 & 5 & 1248 & 4.09 \\
\hline Cingulate gyrus, anterior division & -10 & 40 & 14 & 5 & & 3.8 \\
\hline Precentral & -4 & -34 & 60 & 4 & 929 & 11.1 \\
\hline Subcallosal cortex & -6 & 28 & -20 & 3 & 720 & 3.53 \\
\hline Frontal medial cortex & -4 & 48 & -14 & 3 & & 3.53 \\
\hline Intracalcarine & 8 & -84 & 6 & 2 & 596 & 3.94 \\
\hline Occipital pole $=47$ & -2 & -94 & -2 & 2 & & 3.67 \\
\hline Lingual gyrus & -10 & -82 & -6 & 2 & & 2.83 \\
\hline Temporal fusiform cortex, posterior division & 30 & -36 & -16 & 1 & 266 & 4 \\
\hline Right hippocampus & 24 & -28 & -10 & 1 & & 3.65 \\
\hline Right thalamus & 22 & -32 & 0 & 1 & & 3.21 \\
\hline Temporal occipital fusiform cortex & 30 & -50 & -18 & 1 & & 3.13 \\
\hline Parahippocampal gyrus & 20 & -34 & -10 & 1 & & 2.53 \\
\hline
\end{tabular}

connectivity greater than A-motor connectivity (P-motor $>$ A-motor) (Table 1; Fig. 4). The largest cluster (cluster 5) for A-motor > P-motor extended to both the hemispheres and spanned over from the sensory cortex to primary motor cortex to supplementary motor area and the dorsal anterior cingulate cortex. The other clusters were confined to the right superior temporal gyrus (cluster 4), central opercular cortex and left superior temporal gyrus (cluster 3), and bilateral posterior insula and putamen (clusters 2 and 1). The largest cluster (cluster 6) for P-motor $>$ A-motor extended to both hemispheres and spanned from posterior cingulate gyrus, precuneus, cuneal cortex, angular gyrus, and lateral superior occipital cortex. The other regions spanned by the clusters included paracingulate (cluster 5), precentral gyrus (cluster 4), frontal medial cortex (cluster 3), occipital pole and lingual cortex (cluster 2), and temporal fusiform cortex and parahippocampus (cluster 1).

\section{Discussion}

Our results indicate that human motor cortex contains distinct regions, which, when stimulated, activate different muscle synergies related to pelvic floor muscle control. Moreover, these motor cortical synergy access regions differentially connect with distant and distinct cortical and subcortical areas. Our results are consistent with the idea that cortex modulates lower-level muscle synergies to generate movement patterns (Cheung et al., 2009; Overduin et al., 2012; Bizzi and Cheung, 2013).

Coactivation of the anal sphincter muscle with toe muscles may represent an activation pattern that occurs during posture and locomotion control, which becomes stereotypic such that it also operates even during voluntary contraction with a participant supine. Pelvic floor muscles coactivate with postural muscles to prepare the body for postural changes in an anticipatory fashion (Hodges et al., 2007). One synergy we identified prefer- 
A

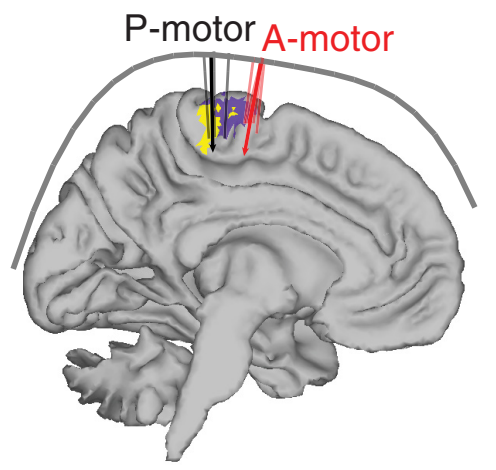

C

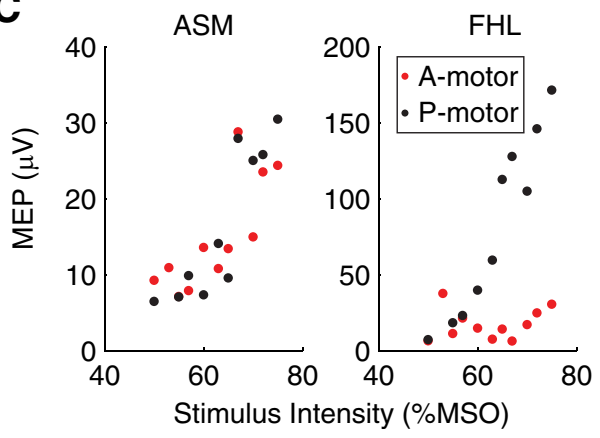

B

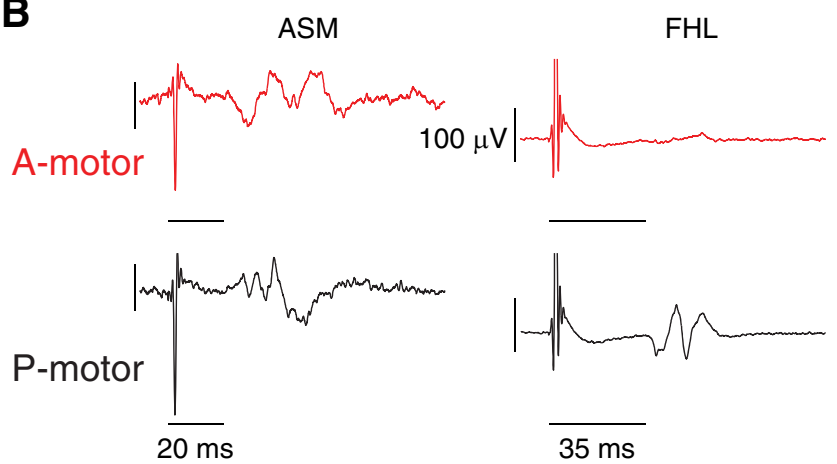

D

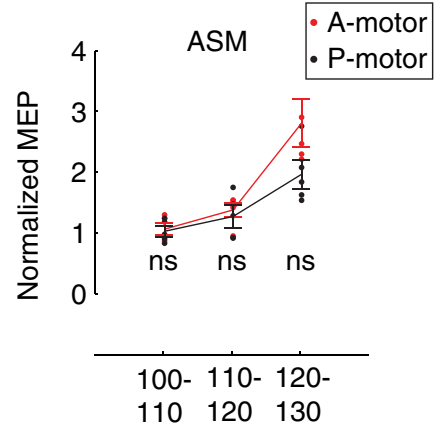

Normalized Stimulus Intensity Bin

(\% resting motor threshold)

Figure 3. TMS evidence that distinct regions of motor cortex, the A-motor and P-motor ROIs, have preferential access to different muscle synergies of anal sphincter muscle (ASM) and FHL muscle control. $\boldsymbol{A}$, We collected MEP from ASM and FHL generated by TMS on the ASM hotspot over the A-motor ROI (A-motor) and FHL hotspot over the P-motor ROI (P-motor). B, Single participant data showing that an MEP was generated in the ASM in response to stimulation of both A-motor and P-motor, whereas an MEP was generated in the FHL only in response to stimulation of $P$-motor and not A-motor. C, Single participant data showing input- output curves for ASM and FHL in response to stimulation of both A-motor and P-motor. Curves indicate that ASM had a similar increase in MEP magnitude with stimulation intensity for both A-motor and P-motor; however, FHL showed a much greater increase in MEP magnitude with stimulation intensity for P-motor than A-motor. $D$, Group data demonstrating the consistent finding that the ASM had a similar increase in MEP with increased stimulation intensity to both A-motor and P-motor, whereas for FHL the increase in MEP magnitude with stimulation intensity was greater in P-motor than A-motor. ${ }^{* *} p<0.01$. ${ }^{* * *} p<0.0001$. Curves indicate average normalized MEP magnitudes in response to normalized stimulation intensity. Errors bars indicate SEM across all participants. Dots indicate original data points from all participants.
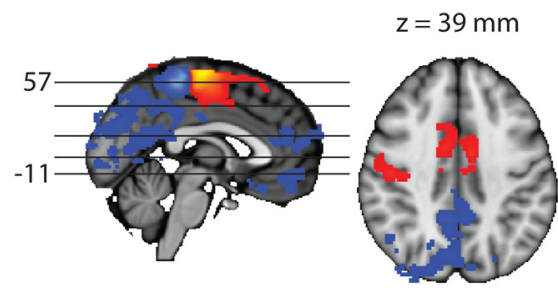

57

$-11$

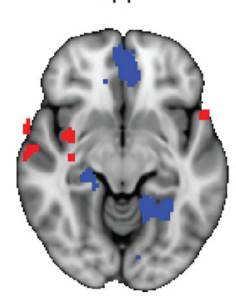

2

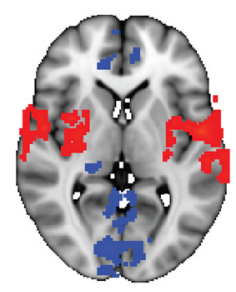

2.3

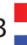

10.1 A-motor > P-motor

11.1 P-motor $>$ A-motor

Figure 4. Resting-statefMRl evidence that the synergyaccess regions in the motor cortex (A-motor and $\mathrm{P}$-motor) have differential functional connectivity with distinct region of brain. A-motor $>\mathrm{P}$-motor (red/ yellow) showed significantly greater functional connectivity $(Z>2.3$; duster significance: $p<0.05$, corrected) with several regions, including bilateral posterior insular cortex, bilateral putamen, and anterior cingulate cortex. P-motor $>$ A-motor (blue/light-blue) showed significantly greater functional connectivity with the regions, including precuneus, posterior cingulate cortex, hippocampus, parahippocampus, lingual cortex, and medial frontal cortex. entially activates pelvic floor muscles and can be used to meet the task demands of maintaining continence while not unnecessarily coactivating postural muscles, which do not themselves contribute to continence (Bø and Stien, 1994). The second synergy we identified promotes automatic contraction of pelvic floor muscles with nonpelvic floor muscles during tasks in which the demand exceeds simply maintaining continence. This flexible coordination of pelvic floor muscles and toe muscles, likely responding to different task demands, makes this synergistic pair of great interest to study the neural mechanisms of synergy control.

In our previous work, we have shown a similar synergistic coupling between pelvic floor muscles and gluteal muscles (Asavasopon et al., 2014). However, in that study, we were unable to assess the specificity of motor cortical connections to the pelvic floor muscles and gluteal muscles because TMS cannot generate reliable MEPs in surface EMG electrodes over gluteal muscles at rest (Fisher et al., 2013). Therefore, switching to a synergistic pair of pelvic floor and toe muscles in this study allowed us to test the specificity of the connection between particular motor cortical regions and their target muscles independent of the motor command because both muscles could be resting during the TMS stimulation. Although EMG and fMRI methods appear to work equally well to characterize the pelvic/gluteal and pelvic/toe synergies, we did not consider gluteal muscles in this study so that all modalities (EMG, fMRI, TMS) could be uniformly applied to the same pair of synergistic muscles. 
Our current work represents a critical step in studying muscle synergies at both the brain and muscle levels in humans. Our framework conceptualizes synergies, as is commonly done in the literature, as a group of muscles with relative activation weightings (encoded in vector $w$ ) under a common time-varying neural drive command (coefficient $c$ ), which can be linearly combined as modules to generate more complex muscle activation patterns (e.g., d'Avella et al., 2003). For the synergies examined in this study, we suggest that regional brain activation in A-Motor $\left(C_{\mathrm{A}-\text { Motor }}\right)$ and P-Motor $\left(C_{\mathrm{P}-\text { Motor }}\right)$ may act as the coefficients weighting the synergies as follows:

$$
\left[\begin{array}{c}
E M G_{\mathrm{ANAL}} \\
E M G_{\mathrm{TOE}}
\end{array}\right]=\left[\begin{array}{c}
w_{1, \mathrm{ANAL}} \\
w_{1, \mathrm{TOE}} \approx 0
\end{array}\right] c_{\mathrm{A}-\text { Motor }}+\left[\begin{array}{c}
w_{2, \mathrm{ANAL}} \neq 0 \\
w_{2, \mathrm{TOE}}
\end{array}\right] c_{\mathrm{P}-\mathrm{Motor}}
$$

Activation of the anal sphincter without associated toe muscle activation suggests that there is a synergy (synergy 1 in the equation) that preferentially activates the anal sphincter compared with the toe $\left(w_{1, \mathrm{TOE}} \approx 0\right)$. From our TMS results, synergy 1 appears to be preferentially accessed by the A-Motor region. Voluntary activation of toe muscles is possible because a second synergy (synergy 2 in the equation) can provide access to the toe muscles, and synergy 2 appears to be activated by the P-Motor region. Our TMS results suggest that P-Motor may activate the anal sphincter as well $\left(w_{2, \text { ANAL }} \neq 0\right)$, suggesting a common motor cortical drive to these muscles. Our functional connectivity findings suggest that the coefficients $C_{\mathrm{A}-\text { Motor }}$ and $C_{\mathrm{P}-\text { Motor }}$ may be governed by activity in distinct brain networks encoding different task demands. A study limitation is that we have not fully shown that anal sphincter and toe muscles fit in the synergy model. Anal sphincter activation was significantly larger during toe muscle activation compared with finger muscle activation: a necessary condition for a synergy. The dynamic muscle activations in our study were based on prior work (Schrum et al., 2011) but were not well suited to analysis of common input to provide sufficient conditions for a synergy at the muscle EMG (Grosse et al., 2002) or brain (Joshua and Lisberger, 2014) level.

Previous research has shown that muscle synergies are likely structured as distinct functional modules in the spinal cord, which can be coordinated by the nervous system to generate a variety of movements (Mussa-Ivaldi et al., 1994a; Kargo and Giszter, 2000; Saltiel et al., 2001; Cheung et al., 2009; Hart and Giszter, 2010). Subcortical mechanisms of muscle activation are clearly essential for automatic reactive motor behaviors of lower limbs. However, there is evidence that motor cortical regions are involved in automatic tasks as well. There is evidence of motor cortical involvement in walking (for review, see Yang and Gorassini, 2006), and in micturition control even without conscious voluntary effort (for review, see Fowler et al., 2008). Motor cortical involvement in automatic motor behaviors may be particularly important when information encoded in higher cortical centers must be rapidly transmitted to override reflexive behaviors. For example, motor cortical areas with direct access to the urethral sphincter muscle are activated during urine storage, presumably to convert information about the social appropriateness of voiding to a protective motor response and delay natural spinal voiding reflexes (Fowler and Griffiths, 2010, Tadic et al., 2010).

Correspondingly, previous studies in primates have hypothesized that automatic muscle synergies may also be structured at the level of M1 by the overlapping representations of different muscles (Rathelot and Strick, 2006). Also, it has been suggested that M1 contains an important anterior/posterior (rostral/caudal) gradient in projections to spinal cord, with an evolutionarily older rostral region without direct cortico-motoneuronal projections ("old" M1), and a evolutionarily newer caudal region displaying cortico-motoneuronal cells ("new M1") (Rathelot and Strick, 2009). We found a complementary gradient with the more anterior A-motor region associated with pelvic floor muscle control and the more posterior P-motor region associated with toe muscle control. Because cortico-motoneuronal cells in $\mathrm{M} 1$ are known to exist for distal hindlimb muscles in nonhuman species (Jankowska et al., 1975; Flament et al., 1992), we hypothesize that the P-motor region may represent a human analog of part of new M1. Because old M1 is more anterior and may be associated with less skilled movements (e.g., pelvic floor contraction to promote continence), we hypothesize that the A-motor region may represent a human analog of part of old M1. However, our current study is limited in that we cannot determine whether the muscle synergies involving pelvic floor and toe muscles are structured at the cortical, subcortical, or spinal level.

To our knowledge, this study is the first to demonstrate how large-scale human brain networks might interact with motor cortex to allow flexible combination of muscle synergies based on task demands. Here, we have shown that the distinct synergy access points in the motor cortex communicate differently with distinct regions of the brain. Previous research suggests that the connectivity patterns we identified may relate to the processing of task demands relevant to the different synergies of pelvic floor muscle control. Insular cortex is considered to be the center of primary viscerosensory representation (Craig, 2011; Kuehn et al., 2015) and has been shown to play an important role in continence control (DasGupta et al., 2007; Tadic et al., 2010, 2012). Insular cortex also plays an important role during voluntary pelvic floor contraction compared with lower limb muscles (Schrum et al., 2011). Precuneus, parahippocampal cortex, hippocampus, and lingual gyrus have been shown to be involved in cognitive aspects of movement in space (Rosenbaum et al., 2004; Epstein, 2008; Schinazi and Epstein, 2010; Wegman and Janzen, 2011; Drew and Marigold, 2015). Based on animal studies, some of these regions appear to be critical for coordinating motor activity during complex motor tasks involving object avoidance, which may involve control of foot placement and toe muscle activity (Andujar et al., 2010).

As recent studies have begun to elucidate the genetic underpinnings of resting-state functional connectivity networks in the human brain (Richiardi et al., 2015), it may be possible to use the approach we have described here to explore developmental versus genetic contributions to muscle synergy structure (Giszter, 2015). Interestingly, as we noted in Results, a small but significant EMG interaction appeared between the anal sphincter and FDI of the hand. Studying extrinsic hand muscles more analogous to the FHL of the toes (such as the flexor digitorum) in future studies might be informative because muscle synergies emerging on the evolutionary time-scale in the forelimb and hindlimb of quadrupeds might display similar interactions with pelvic floor and trunk muscles.

We have recently shown that pelvic floor motor cortical areas have altered resting-state functional connectivity with the posterior insula in men with chronic prostatitis/chronic pelvic pain syndrome (Kutch et al., 2015). Future studies on motor cortical functional connectivity may establish the neural circuitry changes underlying motor cortical excitability and movement 
control changes in chronic pain disorders (Tsao et al., 2008; Hodges and Tucker, 2011).

\section{References}

Andujar JE, Lajoie K, Drew T (2010) A contribution of area 5 of the posterior parietal cortex to the planning of visually guided locomotion: limbspecific and limb-independent effects. J Neurophysiol 103:986-1006. CrossRef Medline

Asavasopon S, Rana M, Kirages DJ, Yani MS, Fisher BE, Hwang DH, Lohman EB, Berk LS, Kutch JJ (2014) Cortical activation associated with muscle synergies of the human male pelvic floor. J Neurosci 34:13811-13818. CrossRef Medline

Baliki MN, Petre B, Torbey S, Herrmann KM, Huang L, Schnitzer TJ, Fields HL, Apkarian AV (2012) Corticostriatal functional connectivity predicts transition to chronic back pain. Nat Neurosci 15:1117-1119. CrossRef Medline

Bernstein NA (1967) The co-ordination and regulation of movements. Oxford: Pergamon.

Bizzi E, Cheung VC (2013) The neural origin of muscle synergies. Front Comput Neurosci 7:51. CrossRef Medline

Bizzi E, Mussa-Ivaldi FA, Giszter S (1991) Computations underlying the execution of movement: a biological perspective. Science 253:287-291. CrossRef Medline

Bø K, Stien R (1994) Needle EMG registration of striated urethral wall and pelvic floor muscle activity patterns during cough, Valsalva, abdominal, hip adductor, and gluteal muscle contractions in nulliparous healthy females. Neurourol Urodyn 13:35-41. CrossRef Medline

Cheung VC, Piron L, Agostini M, Silvoni S, Turolla A, Bizzi E (2009) Stability of muscle synergies for voluntary actions after cortical stroke in humans. Proc Natl Acad Sci U S A 106:19563-19568. CrossRef Medline

Craig AD (2011) Significance of the insula for the evolution of human awareness of feelings from the body. Ann N Y Acad Sci 1225:72-82. CrossRef Medline

DasGupta R, Kavia RB, Fowler CJ (2007) Cerebral mechanisms and voiding function. BJU Int 99:731-734. CrossRef Medline

d'Avella A, Saltiel P, Bizzi E (2003) Combinations of muscle synergies in the construction of a natural motor behavior. Nat Neurosci 6:300-308. CrossRef Medline

Drew T, Marigold DS (2015) Taking the next step: cortical contributions to the control of locomotion. Curr Opin Neurobiol 33:25-33. CrossRef Medline

Drew T, Kalaska J, Krouchev N (2008) Muscle synergies during locomotion in the cat: a model for motor cortex control. J Physiol 586:1239-1245. CrossRef Medline

Epstein RA (2008) Parahippocampal and retrosplenial contributions to human spatial navigation. Trends Cogn Sci 12:388-396. CrossRef Medline

Fisher BE, Lee YY, Pitsch EA, Moore B, Southam A, Faw TD, Powers CM (2013) Method for assessing brain changes associated with gluteus maximus activation. J Orthop Sports Phys Ther 43:214-221. CrossRef Medline

Flament D, Goldsmith P, Lemon RN (1992) The development of corticospinal projections to tail and hindlimb motoneurons studied in infant macaques using magnetic brain stimulation. Exp Brain Res 90:225-228. Medline

Fowler CJ, Griffiths DJ (2010) A decade of functional brain imaging applied to bladder control. Neurourol Urodyn 29:49-55. CrossRef Medline

Fowler CJ, Griffiths D, de Groat WC (2008) The neural control of micturition. Nat Rev Neurosci 9:453-466. CrossRef Medline

Giszter SF (2015) Motor primitives-new data and future questions. Curr Opin Neurobiol 33:156-165. CrossRef Medline

Giszter SF, Mussa-Ivaldi FA, Bizzi E (1993) Convergent force fields organized in the frog's spinal cord. J Neurosci 13:467-491. Medline

Giszter S, Patil V, Hart C (2007) Primitives, premotor drives, and pattern generation: a combined computational and neuroethological perspective. Prog Brain Res 165:323-346. CrossRef Medline

Grosse P, Cassidy MJ, Brown P (2002) EEG-EMG, MEG-EMG and EMGEMG frequency analysis: physiological principles and clinical applications. Clin Neurophysiol 113:1523-1531. CrossRef Medline

Hart CB, Giszter SF (2010) A neural basis for motor primitives in the spinal cord. J Neurosci 30:1322-1336. CrossRef Medline

Hodges PW, Tucker K (2011) Moving differently in pain: a new theory to explain the adaptation to pain. Pain 152:S90-S98. CrossRef Medline
Hodges PW, Sapsford R, Pengel LH (2007) Postural and respiratory functions of the pelvic floor muscles. Neurourol Urodyn 26:362-371. CrossRef Medline

Jankowska E, Padel Y, Tanaka R (1975) Projections of pyramidal tract cells to alpha-motoneurones innervating hind-limb muscles in the monkey. J Physiol 249:637-667. CrossRef Medline

Joshua M, Lisberger SG (2014) A framework for using signal, noise, and variation to determine whether the brain controls movement synergies or single muscles. J Neurophysiol 111:733-745. CrossRef Medline

Kargo WJ, Giszter SF (2000) Rapid correction of aimed movements by summation of force-field primitives. J Neurosci 20:409-426. Medline

Kuehn E, Mueller K, Lohmann G, Schuetz-Bosbach S (2015) Interoceptive awareness changes the posterior insula functional connectivity profile. Brain Struct Funct. Advance online publication. Retrieved Jan. 23, 2015. doi:10.1007/s00429-015-0989-8. CrossRef Medline

Kurusu K, Kitamura J (1999) Long-latency reflexes in contracted hand and foot muscles and their relations to somatosensory evoked potentials and transcranial magnetic stimulation of the motor cortex. Clin Neurophysiol 110:2014-2019. CrossRef Medline

Kutch JJ, Yani MS, Asavasopon S, Kirages DJ, Rana M, Cosand L, Labus JS, Kilpatrick LA, Ashe-McNalley C, Farmer MA, Johnson KA, Ness TJ, Deutsch G, Harris RE, Apkarian AV, Clauw DJ, Mackey SC, Mullins C, Mayer EA (2015) Altered resting state neuromotor connectivity in men with chronic prostatitis/chronic pelvic pain syndrome: a MAPP Research Network Neuroimaging Study. Neuroimage Clin 8:493-502. CrossRef Medline

Lefaucheur JP (2005) Excitability of the motor cortical representation of the external anal sphincter. Exp Brain Res 160:268-272. CrossRef Medline

Mussa-Ivaldi FA, Giszter SF, Bizzi E (1994b) Linear combinations of primitives in vertebrate motor control. Proc Natl Acad Sci U S A 91:75347538. CrossRef Medline

Overduin SA, d'Avella A, Carmena JM, Bizzi E (2012) Microstimulation activates a handful of muscle synergies. Neuron 76:1071-1077. CrossRef Medline

Overduin SA, d'Avella A, Carmena JM, Bizzi E (2014) Muscle synergies evoked by microstimulation are preferentially encoded during behavior. Front Comput Neurosci 8:20. CrossRef Medline

Pelliccioni G, Scarpino O, Piloni V (1997) Motor evoked potentials recorded from external anal sphincter by cortical and lumbo-sacral magnetic stimulation: normative data. J Neurol Sci 149:69-72. CrossRef Medline

Rathelot JA, Strick PL (2006) Muscle representation in the macaque motor cortex: an anatomical perspective. Proc Natl Acad Sci U S A 103:82578262. CrossRef Medline

Rathelot JA, Strick PL (2009) Subdivisions of primary motor cortex based on cortico-motoneuronal cells. Proc Natl Acad Sci U S A 106:918-923. CrossRef Medline

Richiardi J, Altmann A, Milazzo AC, Chang C, Chakravarty MM, Banaschewski T, Barker GJ, Bokde AL, Bromberg U, Büchel C, Conrod P, Fauth-Bühler M, Flor H, Frouin V, Gallinat J, Garavan H, Gowland P, Heinz A, Lemaitre H, Mann KF, et al. (2015) Correlated gene expression supports synchronous activity in brain networks. Science 348:1241-1244. CrossRef Medline

Rosenbaum RS, Ziegler M, Winocur G, Grady CL, Moscovitch M (2004) "I have often walked down this street before": fMRI studies on the hippocampus and other structures during mental navigation of an old environment. Hippocampus 14:826-835. CrossRef Medline

Roy AK, Shehzad Z, Margulies DS, Kelly AM, Uddin LQ, Gotimer K, Biswal BB, Castellanos FX, Milham MP (2009) Functional connectivity of the human amygdala using resting-state fMRI. Neuroimage 45:614-626. CrossRef Medline

Saltiel P, Wyler-Duda K, D’Avella A, Tresch MC, Bizzi E (2001) Muscle synergies encoded within the spinal cord: evidence from focal intraspinal NMDA iontophoresis in the frog. J Neurophysiol 85:605-619. Medline

Sapsford R, Stafford R, Hodges P (2010) How does contraction of lumbrical muscles of the foot reduce the urge to void? In: Joint Annual Meeting of the International Continence Society and the International Urogynecological Association, Toronto.

Schinazi VR, Epstein RA (2010) Neural correlates of real-world route learning. Neuroimage 53:725-735. CrossRef Medline 
Schrum A, Wolff S, van der Horst C, Kuhtz-Buschbeck JP (2011) Motor cortical representation of the pelvic floor muscles. J Urol 186:185-190. CrossRef Medline

Tadic SD, Griffiths D, Murrin A, Schaefer W, Aizenstein HJ, Resnick NM (2010) Brain activity during bladder filling is related to white matter structural changes in older women with urinary incontinence. Neuroimage 51:1294-1302. CrossRef Medline

Tadic SD, Griffiths D, Schaefer W, Murrin A, Clarkson B, Resnick NM (2012) Brain activity underlying impaired continence control in older women with overactive bladder. Neurourol Urodyn 31:652-658. CrossRef Medline

Ting LH, Macpherson JM (2005) A limited set of muscle synergies for force control during a postural task. J Neurophysiol 93:609-613. CrossRef Medline

Torres-Oviedo G, Macpherson JM, Ting LH (2006) Muscle synergy organization is robust across a variety of postural perturbations. J Neurophysiol 96:1530-1546. CrossRef Medline
Tsao H, Galea MP, Hodges PW (2008) Reorganization of the motor cortex is associated with postural control deficits in recurrent low back pain. Brain 131:2161-2171. CrossRef Medline

Turnbull GK, Hamdy S, Aziz Q, Singh KD, Thompson DG (1999) The cortical topography of human anorectal musculature. Gastroenterology 117: 32-39. CrossRef Medline

Waters-Metenier S, Husain M, Wiestler T, Diedrichsen J (2014) Bihemispheric transcranial direct current stimulation enhances effectorindependent representations of motor synergy and sequence learning. J Neurosci 34:1037-1050. CrossRef Medline

Wegman J, Janzen G (2011) Neural encoding of objects relevant for navigation and resting state correlations with navigational ability. J Cogn Neurosci 23:3841-3854. CrossRef Medline

Yang JF, Gorassini M (2006) Spinal and brain control of human walking: implications for retraining of walking. Neuroscientist 12:379-389. CrossRef Medline 